\title{
Una reflexión acerca de las prisiones privadas: ¿pragmatismo o ideología?
}

\author{
Albert Pedrosa \\ Universitat Autònoma de Barcelona \\ albert.pedrosa@uab.cat
}

Recepción: 01-11-2018

Aceptación: 08-03-2019

Publicación: 29-04-2019

\section{Resumen}

Este texto pertenece a una mesa redonda que ofrece un debate con James B. Jacobs, autor del clásico de la sociología de las prisiones Stateville: The penitentiary in mass society, sobre la vigencia de su obra cuarenta años después. Este comentario presenta una reflexión acerca de las razones que han podido llevar al surgimiento de las prisiones privadas, confrontando argumentos que defienden su surgimiento por razones pragmáticas con otros que las sitúan como producto de la expansión hacia el castigo de la ideología neoliberal. Por último, se realiza una breve reflexión acerca de la posibilidad de que en el futuro este tipo de instituciones lleguen al contexto español.

Palabras clave: prisión; castigo; neoliberalismo; pragmatismo; ideología

Abstract. A reflection on private prisons: pragmatism or ideology?

This text forms part of a round table discussion with James B. Jacobs, author of the classic study on the sociology of prisons Stateville: The Penitentiary in Mass Society, and examines the validity of his work 40 years later. The commentary presents some thoughts on the reasons that have led to the emergence of private prisons. Arguments that defend privately operated penal facilities for pragmatic reasons are contrasted against those that view them as a product of an expanding neoliberal ideology towards punishment. Finally, a brief reflection is made about the possibility of introducing this kind of institutions in Spanish in the near future.

Keywords: prison; punishment; neoliberalism; pragmatism; ideology 


\section{Sumario}

\author{
1. La gestión de las cárceles \\ "post-Stateville»; nuevas tendencias \\ e influencias culturales \\ 2. Pragmatismo
}

3. Ideología

4. Reflexiones finales: ¿se privatizarán las prisiones españolas?

Referencias bibliográficas

\section{La gestión de las cárceles «post-Stateville»; nuevas tendencias e influencias culturales}

En la obra de James B. Jacobs, uno de los temas centrales es el proceso que ha llevado a la prisión a convertirse en una institución permeable a las influencias sociohistóricas y culturales, mostrando que, aunque la prisión pueda tener características y formas de funcionar propias que la asemejan a una sociedad particular (Sykes, 1958), esta también se ve afectada por su contexto y los valores que las personas importan desde fuera hacia dentro de la institución (Jacobs, 1977). Uno de los puntos clave del libro es la descripción de los procesos que llevaron a pasar de una autoridad —en la gestión y dirección de la prisión - autoritaria a una de tipo legal burocrática, en un análisis profundo que muestra cómo la influencia de los movimientos sociales, el contexto histórico, pero también las prácticas culturales y de las organizaciones, pueden afectar al modo de articular el castigo en las cárceles.

Sin embargo, y una vez superado el período de expansión punitiva en que se enmarca el libro, resulta imprescindible explorar cómo la nueva modernidad ha permeado dentro de las cárceles, especialmente en la época de la globalización, la cultura de masas y la expansión de las políticas neoliberales y de las lógicas del mercado global (en una línea parecida, ver el texto de Ignacio González Sánchez en este mismo debate). Sobre dichas cuestiones han coexistido dos grandes perspectivas: la defendida por David Garland (2001), quien propone la existencia — a partir de la segunda mitad del siglo $\mathrm{xX}$ - de una "cultura del control» compartida y expandida, especialmente en EE. UU. y el Reino Unido; y aquellos autores que han puesto en duda esta uniformidad en el castigo, destacando las particularidades de las políticas penales y las diferencias en las culturas del castigo entre distintos países (Lacey, 2010).

Por lo tanto, en la actualidad es relevante ver si —y cómo— la expansión moderna de las políticas neoliberales ha afectado a la institución del castigo, y en qué medida esto puede variar en diferentes contextos centrándonos, además, en cómo dichas influencias culturales han afectado al gobierno y las formas de gestión de los centros penitenciarios, en la línea del libro discutido. Por esta razón en este texto reflexionaré brevemente acerca de un fenómeno particular, el de las cárceles privadas, confrontando diversos argumentos acerca de su surgimiento y relación con la expansión de las políticas neoliberales, así como planteando si algún día veremos este tipo de instituciones en España. 


\section{Pragmatismo}

En este texto, cuando me refiera a prisiones privadas hablaré de aquellas cuya gestión ha sido derivada a empresas privadas por parte del Estado, tema que también fue objeto de debate con el autor. Por lo tanto y debido a la limitación de espacio, no será objeto de esta reflexión la privatización de ciertos servicios (como podrían ser la alimentación o la limpieza) vinculados a la actividad penitenciaria, sino que me centraré en la privatización de la dirección y gestión de los centros, la cual tiene mayor incidencia en el mantenimiento de la seguridad interior y en las condiciones de vida de las personas que cumplen condena. Aunque el caso de Estados Unidos ha sido el más analizado y a menudo el presentado como representativo de este fenómeno, estos centros también pueden encontrarse en el Reino Unido o en Australia, incluso con un mayor uso y desarrollo ${ }^{1}$. Este tipo de instituciones tuvieron su auge a partir de la segunda mitad del siglo XX, especialmente en la década de los sesenta, coincidiendo con un gran crecimiento de la población penitenciaria en EE. UU. que conllevó la necesidad de construir más prisiones, y rápido, tarea que se confió al sector privado (Jones y Newburn, 2005, entre otros).

Aunque estas instituciones han acabado absorbiendo una pequeña parte del total de personas encarceladas por el sistema ${ }^{2}$, han sido objeto de amplios debates debido a las implicaciones que puede comportar el hecho de trasladar el control y la responsabilidad de una institución tan importante como es el castigo del Estado a una entidad privada. Los principales argumentos esgrimidos por los defensores de dichas instituciones se han centrado en su carácter pragmático, ya que estas pueden ser más económicas y, además, al no estar sujetas a la burocracia estatal, permiten una mayor flexibilidad en la contratación de personal e implementación de nuevas y mejores formas de castigar. Así lo defendía el propio James Jacobs (2018) en el debate: «Los emprendedores que piensan que pueden gestionar las prisiones mejor y de forma más económica y crear mejores condiciones para vivir el encarcelamiento y de remuneración tienen todos los incentivos para hacer un buen trabajo».

Sin embargo, la literatura ha puesto en duda algunos de estos argumentos de tipo pragmático, puesto que las evaluaciones llevadas a cabo sobre el tema concluyen que no se puede afirmar que las prisiones privadas supongan un ahorro significativo con respecto a las de gestión pública (Perrone y Pratt, 2003; Pratt y Maahs, 1999); ni tampoco parece que su gestión en el día a día se pueda catalogar como más eficiente. Al contrario, numerosos autores han destacado que este tipo de instituciones pueden suponer costes adicionales para las personas encarceladas en cuanto a las condiciones de su encierro o generar

1. La expansión de este tipo de instituciones o prácticas de privatización se ha producido también en Sudáfrica y algunos países de América Latina y Asia, aunque con menor desarrollo (Mason, 2013).

2. Aproximadamente un $8 \%$ del total de personas encarceladas en EE. UU. y un $14 \%$ en el Reino Unido. Las cifras más altas se encuentran en Australia, donde rondan el $20 \%$ (Mason, 2013). 
problemas con el mantenimiento del orden y la seguridad (Camp y Gaes, 2002), y que los costes parecen depender más de las características particulares de cada centro (antigüiedad, número de internos o tamaño) que del modelo concreto de gestión (Perrone y Pratt, 2003).

Siguiendo con lo anterior, no solo no se puede afirmar que las prisiones privadas supongan un ahorro real con respecto a las públicas, sino que además han supuesto - en su aplicación práctica - una excusa para la incorporación de dinámicas neoliberales en la gestión y contratación dentro de la institución. En un interesante trabajo, Brett Burkhardt (2017) recopiló y analizó datos de EE. UU. para responder a dos preguntas: a quién encerramos en las prisiones privadas y quién trabaja en ellas. Los resultados al respecto son claros: en estas instituciones se encierra a más personas pertenecientes a minorías y con penas cortas con el objetivo de que más «usuarios» pasen por el sistema y, así, recibir mayores subvenciones por número de presos. Por otro lado, y a diferencia de las prisiones de gestión pública, las instituciones privadas tienen menos personal y con salarios más bajos y emplean en mayor medida a personas de minorías y a mujeres. Así pues, esta mayor flexibilización y privatización de la gestión ha acabado afectando a los derechos y las condiciones de las personas pertenecientes a las minorías que están encerradas, pero también a aquellas que trabajan en estos centros. Sin embargo, esto también podría variar entre países, ya que existen otros autores que han mostrado que los funcionarios no siempre ven necesariamente mal este tipo de gestión (Mclean y Liebling, 2008).

\section{Ideología}

Anteriormente mostrábamos que, aunque existe un debate abierto acerca de las razones de tipo pragmático empleadas por los defensores de las prisiones privadas, finalmente la literatura ha puesto en duda que dichas instituciones supongan un ahorro real o sean más eficientes, por lo que debemos preguntarnos por qué tanto en EE. UU. como en el Reino Unido surgieron dichas instituciones obviando otras alternativas, y por qué dichos procesos de privatización se han mantenido hasta hoy día. Al respecto, coincido con Jones y Newburn (2005) cuando defienden que el surgimiento de este fenómeno en parte se debe más a motivos ideológicos que pragmáticos, arropados por intereses de ciertos grupos de presión en EE. UU., y como expresión de los ideales neoliberales del gobierno de Margaret Thatcher en el Reino Unido. Por lo tanto, vemos que la expansión de los ideales neoliberales que ha tenido lugar en algunos países puede haber afectado de formas diversas a sus instituciones y puede haber provocado el surgimiento de la privatización de las prisiones, de la cual nos queda también por conocer cómo ha afectado a su gestión o a otras instituciones anexas (González Sánchez, 2015), como el mercado laboral o las familias.

Hemos visto que, aunque la implantación de las cárceles privadas se ha defendido desde el pragmatismo y la promesa de una gestión más eficaz, dicha eficacia no parece haberse traducido en una realidad, ya que este tipo de instituciones son una expresión de intereses y posicionamientos ideológicos con- 
cretos. Por lo tanto, y siguiendo algunas de las ideas presentadas en Stateville, no parecería descabellado sostener que el auge y la expansión de las políticas neoliberales y la lógica de mercado - más marcada en los países ya mencionados- pueden ser en parte responsables del surgimiento de las prisiones privadas en detrimento de otro tipo de alternativas. Además, esto tendría importantes implicaciones en cómo estas influencias pueden afectar al tipo de gestión y autoridad que se aplica en las cárceles; por ejemplo, llevando los ideales y las prácticas de la empresa privada al interior de las prisiones, priorizando el ahorro de costes en detrimento de la seguridad de los internos, pero también afectando a las condiciones laborales de los trabajadores, con importantes efectos para su moral laboral (ver el texto de Cristina Güerri Ferrández en este mismo debate). Así pues, esta privatización habría acabado afectando al modo de gestionar las prisiones no en el sentido esperado — mayor innovación y menor burocraciasino con la aplicación de la lógica empresarial, tal como también admite Jacobs (2018): «No creo que puedas hacer un buen trabajo y barato al mismo tiempo [...] mucha de la responsabilidad es del gobierno por no invertir los recursos necesarios, obtienes lo que pagas».

\section{Reflexiones finales: ¿̇e privatizarán las prisiones españolas?}

Por último, me gustaría terminar el texto con una reflexión breve - y limitada por el espacio- acerca de la posibilidad de que algún día veamos este tipo de instituciones en España. El contexto español se caracteriza por un uso desproporcionado del encarcelamiento aun cuando su volumen de delincuencia es relativamente bajo (Díez Ripollés, 2006a). A pesar de que todavía hoy las causas que afectan al ascenso y descenso del encarcelamiento en España son algo difusas, estas se han solido atribuir a la especial dureza con que el legislador y el sistema penal han tratado los delitos relacionados con las drogas y la delincuencia patrimonial, y al incremento de la duración de las penas (Almeda, 2005; Almeda, Di Nella y Navarro, 2011; Cid, 2008; Cid y Larrauri, 2009; Díez Ripollés, 2006b; Pedrosa, 2018; entre otros). Sin embargo, otras voces han puesto de manifiesto que dichas explicaciones, muchas veces ancladas en la tradición jurídica que caracteriza a la criminología española, serían insuficientes en tanto que simplifican un fenómeno social más amplio y complejo (González Sánchez, 2011) y olvidan el papel que otras instituciones y agentes puedan estar desempeñando.

Sin entrar en dicho debate, sí resulta paradójico que en un país con cifras tan elevadas de encarcelamiento no se haya planteado de forma sólida el debate sobre la privatización del castigo. En este sentido, más allá de la privatización de ciertos servicios asociados con el funcionamiento de los centros, no encontramos en España instituciones penales para adultos privadas o con su gestión privatizada. Sí encontramos en la justicia de menores un grado de privatización de sus centros, que no son de gestión pública, pero por ley estos no pueden ser gestionados por entidades con ánimo de lucro, por lo que nos encontramos ante un modelo mixto que, sin ser público, no puede estar sujeto a las críticas 
que se mencionaban anteriormente respecto a los centros privados y su gestión en la lógica de mercado ${ }^{3}$.

Esto ineludiblemente nos lleva a plantear que quizás esto no se deba a una falta de tendencias punitivas, sino a determinadas influencias de nuestro contexto cultural y a la lógica y las características de la organización del Estado español, que han podido ejercer como factores protectores frente a la privatización del castigo. Así pues, algunos autores han defendido las particularidades de los estados del bienestar del sur de Europa (entre ellos, España) destacando la importante protección del cuerpo de trabajadores del sector público, el hecho de que algunos países estuvieron bajo el yugo de gobiernos dictatoriales hasta la historia reciente, o la importancia de la Iglesia, entre otros factores distintivos (Moreno, 2001). A modo de ejemplo, recientemente Ibàñez y Pedrosa (2018) han defendido que algunas características propias del contexto cultural español, en concreto el familiarismo y los valores tradicionales asociados a la familia, son transmitidas a través de distintas instituciones, entre ellas, la prisión. Todos estos factores pueden haber reforzado la protección y la importancia del carácter público del castigo, sujeto a los principios y las garantías del estado democrático. Aun así, existen otras razones que también pueden tener influencia, entre ellas, que la reeducación social de los presos esté constitucionalmente reconocida, o la importancia que las entidades del tercer sector tienen en la reinserción postpenitenciaria (Cid e Ibàñez, 2018). En síntesis, quizás todos estos factores hayan ayudado a crear un clima social y cultural en el cual el castigo se vincula fuertemente al Estado, sin plantear el traspaso de políticas neoliberales, incluso en tiempos de crisis, hacia dicha institución, por lo que el castigo y su gestión se seguirían viendo como un asunto público y social. Por lo tanto, aunque es complejo aventurar si en el futuro veremos prisiones privadas en España, sí podemos aventurar que este tipo de instituciones encontrarán obstáculos y dificultades debido a la influencia del contexto cultural y modelo de estado, puesto que las ideas expuestas por Jacobs en Stateville siguen hoy vigentes y nos recuerdan la importancia de analizar cómo el contexto social que envuelve a la prisión puede afectar a su gestión y evolución.

\section{Referencias bibliográficas}

AlmedA, Elisabet (2005). «Women’s imprisonment in Spain». Punishment \& Society, 7, (2), 183-199. $<$ https://doi.org/10.1177/1462474505050442>

Almeda, Elisabet; Di Nella, Dino; NAVARro, Carmen (2011). «Mujeres, cárceles y drogas: datos y reflexiones». Oñati Socio-Legal Series, 2 (6), 122-45.

BURKHARDT, Brett. C. (2017). "Who is in private prisons? Demographic profiles of prisoners and workers in American private prisons». International Journal of Law, Crime and Justice, 51, 24-33.

<https://doi.org/10.1016/j.ijlcj.2017.04.004>

3. En todo caso, otro fenómeno que habría que estudiar sería si este modelo tiene un impacto diferenciado en las condiciones laborales de los trabajadores de los centros. 
CAMP, Scott D. y GAES, Gerald G. (2002). «Growth and quality of US private prisons: Evidence from a national survey». Criminology \& Public Policy, 1 (3), 427-450. <https://doi.org/10.1111/j.1745-9133.2002.tb00102.x>

CID, José (2008). «El incremento de la población reclusa en España entre 1996-2006: Diagnóstico y remedios». Revista Española de Investigación Criminológica, 6, 1-31.

CID, José y LARRAURI, Elena (2009). «Development of crime, social change, mass media, crime policy, sanctioning practice and their impact on prison population rates». Sistema Penal \& Violência, Porto Alegre, 1 (1), 1-21.

CiD, José e IBĀÑEZ, Aina. (2018). «Prisoner resettlement in Spain - Good practices for early-released prisoners and prisoners lost in transition that fully serve their sentence». En: Frieder Dünkel, Ineke Pruin, Anette Storgaard y Jonas Weber (eds.). Prisoner Resettlement in Europe. Routledge.

Díez Ripollés, José Luis (2006a). "Algunos rasgos de la delincuencia en España a comienzos del siglo XXI». Revista Española de Investigación Criminológica, 4, 1-19.

- (2006b). «La evolución del sistema de penas en España: 1975-2003». Revista Electrónica de Ciencia Penal y Criminología, 8 (7), 1-25.

GARLAND, David (2001). The culture of control. Crime and social order in contemporary society. Oxford: Oxford University Press.

GonZÁlez SÁnCHEZ, Ignacio (2011). «Aumento de presos y Código Penal: una explicación insuficiente». Revista Electrónica de Ciencia Penal y Criminología, 13 (4).

- (2015). «Neoliberalismo y expansión del sistema penal: apuntes sobre una relación no anunciada». Encrucijadas-Revista Crítica de Ciencias Sociales, 9.

IBÀNEZ, Aina y PEDrosA, Albert (2018). «Cárcel y familiarismo: ¿Usamos a las familias como agencias de reinserción?». Encrucijadas-Revista Crítica de Ciencias Sociales, 16.

Jacobs, James B. (1977). Stateville. The Penitentiary in Mass Society. Londres: The University of Chicago Press.

- (2018). «Author meets critics: Stateville Revisited». III Conferencia Internacional del Máster en Criminología y Ejecución Penal Barcelona, 11 de mayo.

Jones, Trevor y Newburn, Tim (2005). «Comparative Criminal Justice PolicyMaking in the United States and the United Kingdom: The Case of Private Prisons». British Journal of Criminology, 45 (1), 58-80. <https://doi.org/10.1093/bjc/azh067>

LACEY, Nicola (2010). «Differentiating among penal states». The British Journal of Sociology, 61 (4), 778-94. <https://doi.org/10.1111/j.1468-4446.2010.01341.x>

MASON, Cody. (2013). International growth trends in prison privatization. Washington, DC: Sentencing Reform Project.

Mclean, Clare y Liebling, Alison (2008). «Prison staff in the public and private sector». En: Jamie Bennett, Ben Crewe y Azrini Wahidin (eds.). Understanding prison staff. Londres: Willan, 92-114.

MorenO, Luis (2001). «La "vía media" española del modelo de bienestar mediterráneo». Papers. Revista de Sociologia, 63, 67-82. <http://dx.doi.org/10.5565/rev/papers/v63n0.1207>

Pedrosa, Albert (2018). «¿Discrimina el Código Penal español a las mujeres?». Revista Española de Investigación Criminológica, 16, 1-22.

Perrone, Dina y PratT, Travis C. (2003). "Comparing the quality of confinement and cost-effectiveness of public versus private prisons: What we know, why we do not know more, and where to go from here». The Prison Journal, 83 (3), 301-22. <https://doi.org/10.1177/0032885503256329> 
PrATT, Travis C. y MAAHS, Jeff (1999). «Are private prisons more cost-effective than public prisons? A meta-analysis of evaluation research studies». Crime \& Delinquency, 45 (3), 358-71.

<https://doi.org/10.1177/0011128799045003004>

SYKES, Gresham M. (1958). The society of captives: A study of a maximum security prison. Princeton: Princeton University Press. 